\title{
Strategy for Stimuli-Induced Spin Control Using a Liquescent Radical Cation
}

\author{
Shuichi Suzuki,* Ryochi Maya, Yoshiaki Uchida, Takeshi Naota*
}

Graduate School of Engineering Science, Osaka University, 1-3 Machikaneyama, Toyonaka, Osaka 560-8531, Japan 


\section{Table of Contents}

Photographs of $\mathbf{1}^{\cdot+} \cdot \mathbf{N T f}_{2}^{-}$in the different states (Figure S1) p. S2

Thermogravimetry and differential scanning calorimetry curves (Figure S2) p. S3

$\begin{array}{ll}\text { Calculated structures of } \mathrm{NTf}_{2}^{-}(\text {Figure } \mathrm{S} 3) & \text { p. S4 }\end{array}$

Temperature dependency of ESR intensity

from the PM-OS to DM-GS states (Figure S4) p. S5

ESR spectra changes at variable temperature (Figure S5) p. S6

$\begin{array}{ll}\text { Temperature dependence of } \chi_{\mathrm{p}} \text { for } \mathbf{1}^{\mathbf{}^{+}} \mathbf{N T T f}_{2}^{-}(\text {Figure S6) } & \text { p. S7 }\end{array}$

UV/vis/near-IR spectra of $\mathbf{1}^{\bullet+} \cdot \mathbf{N T f}_{2}^{-}($Figure S7) $\quad$ p. S8

Detailed dimer structure of $\mathbf{1}^{*+}$ in the DM-GC state (Figure S8) p. S9

Packing structure of $\mathbf{1}^{\cdot+} \cdot \mathbf{N T f}_{\mathbf{2}}^{-}$in the DM-GC state (Figure S9) p. S10

Powder XRD patterns at the phase transition

from the PM-OS to DM-GS states (Figure S10) p. S11

Powder XRD patterns in the DM-GC and DM-GS states (Figure S11) p. S12

Proposed mechanism of the changes in radical associates (Figure S12) p. S13

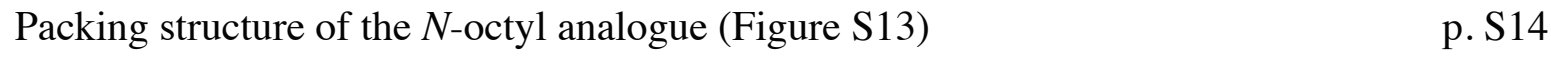

ESR and UV/vis/near-IR spectra of the $N$-octyl analogue (Figure S14) p. S15

Calculated exchange interactions of the intermolecular contacts

in the dimeric pairs of $\mathbf{1}^{\cdot+}$ and the $N$-octyl analogue (Table S1) p. S16

Packing diagram and dimer structures in

a polymorph crystal of $\mathbf{1}^{+} \cdot \mathbf{N T f}_{2}^{-}($Figure S15) p. S17

$\begin{array}{ll}\text { Results of TDDFT calculations (Figure S16) p. S18 } & \text { p) }\end{array}$

$\begin{array}{lr}\text { Crystallographic data (Table S2) } & \text { p. S19 }\end{array}$

$\begin{array}{ll}\text { References } & \text { p. } S 20\end{array}$ 
a)

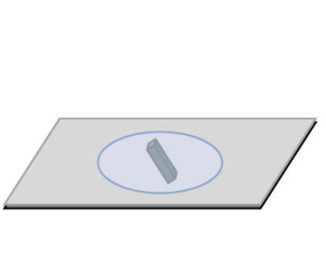

c)

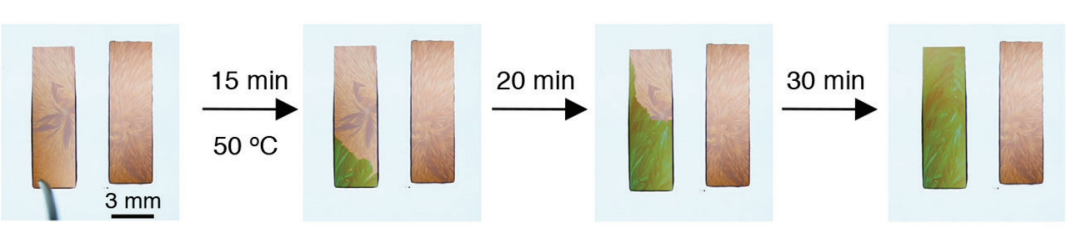

b)

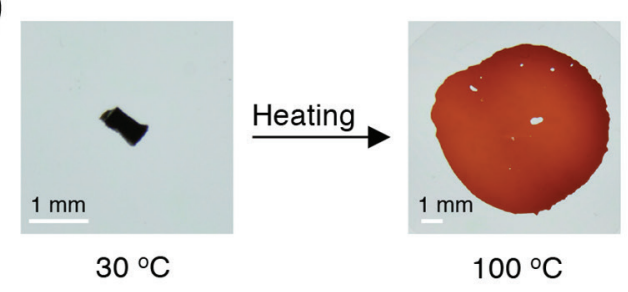

Figure S1. a) Schematic figure of the sample for the photographs for Figure 1, and Movies S1, S2. The sample was placed on a slide glass and it was covered with a thin-glass plate (thickness 0.12$0.17 \mathrm{~mm}$ ) for taking photographs and movies under thin layer conditions. The slide glass with the sample was warmed by a heater with appropriate temperature. b) Photographs in the DM-GC (the crystal size: ca. $0.8 \times 0.4 \times 0.2 \mathrm{~mm}^{3}$ ) and PM-OL states (diameter: ca. $8 \mathrm{~mm}$; thickness: ca. $1-2 \mu \mathrm{m}$ ). c) Photographs of changes from the PM-OS to DM-GS states with (left) and without (right) mechanical stress using a preheated steel needle (see also, Movie S3). The conditions of the samples were similar to that of Figure S1a except for using rectangle-shape cover glass. 

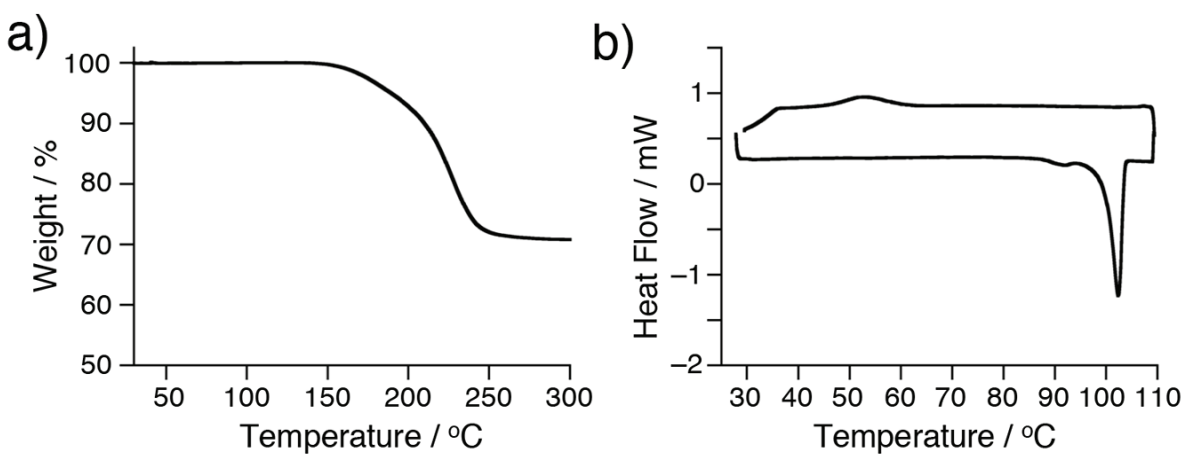

Figure S2. Thermogravimetry (a; scan rate: $2 \mathrm{~K} / \mathrm{min}$ ) and differential scanning calorimetry (b; scan rate: $2 \mathrm{~K} / \mathrm{min}$ ) curves of $\mathbf{1}^{\cdot+} \cdot \mathbf{N T f}_{2}^{-}$. The thermogravimetric analysis revealed no weight loss until $150{ }^{\circ} \mathrm{C}$ under aerated conditions, demonstrating that the phase transition from the DM-GC to PMOL states occurred without any decomposition. Differential scanning calorimetry revealed one endothermic peak at $103{ }^{\circ} \mathrm{C}$ during the heating process and one weak and broad exothermic peak at $60{ }^{\circ} \mathrm{C}$ during the cooling process, assignable to the phase transitions from the DM-GC to PM-OL states and from the PM-OL to PM-OS states, respectively. The peak corresponding to the transformation from the PM-OS to DM-GS states was not observed because the phase transition proceeded slowly in the solid state. The samples in the DM-GS state which was prepared from the melted state (PM-OL) by long standing at room temperature showed the similar diagrams. The similar diagrams were observed in the cases of much slower scan rates. 


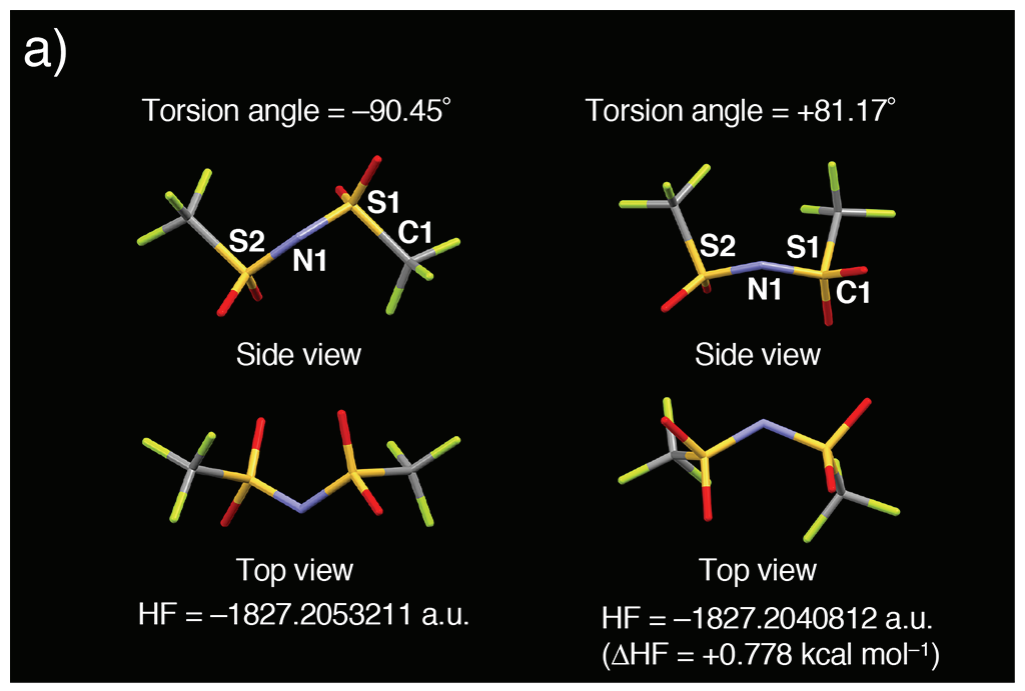

b)

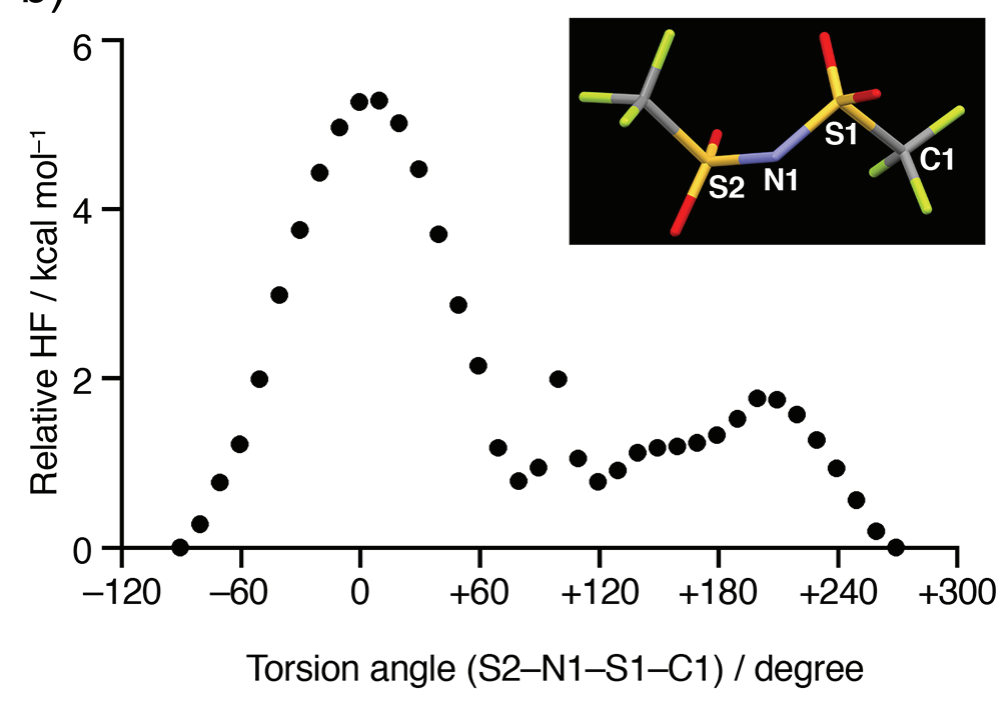

Figure S3. (a) Two kinds of optimized structure of the $\mathrm{NTf}_{2}^{-}$calculated from the geometry of the crystal structure by Gaussian 09 at the b3lyp/6-31g** level of theory. ${ }^{\mathrm{S} 1}$ Although the torsion angles defined by S2-N1-S1-C1 were different, the heat of formations (HF) were very similar. The HF of the left conformer was slightly stable than that of the right one. (b) The relative HF depended on the torsion angles defined by $\mathrm{S} 2-\mathrm{N} 1-\mathrm{S} 1-\mathrm{C} 1$. In this calculations, the torsion angles were feezed at corresponding angles (10 degree intervals from the most stable optimized structure) and other coordinates were optimized by Gaussian 09 at the b3lyp/6-31g** level of theory. ${ }^{\text {S1 }}$ The differences of the HFs for the conformers were estimated to be only within $6 \mathrm{kcal} \mathrm{mol}^{-1}$, which suggested that $\mathrm{NTf}_{2}^{-}$possessed high flexibility. 
a)

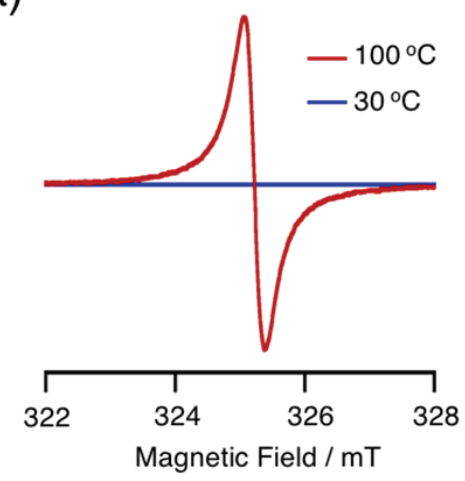

b)

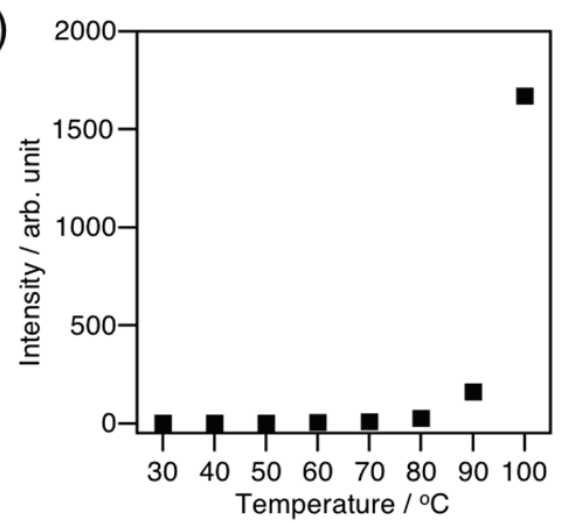

Figure S4. a) ESR spectra at $30{ }^{\circ} \mathrm{C}$ (blue line, DM-GC) and $100{ }^{\circ} \mathrm{C}$ (red line, PM-OL). b) Temperature dependency of ESR intensities. 

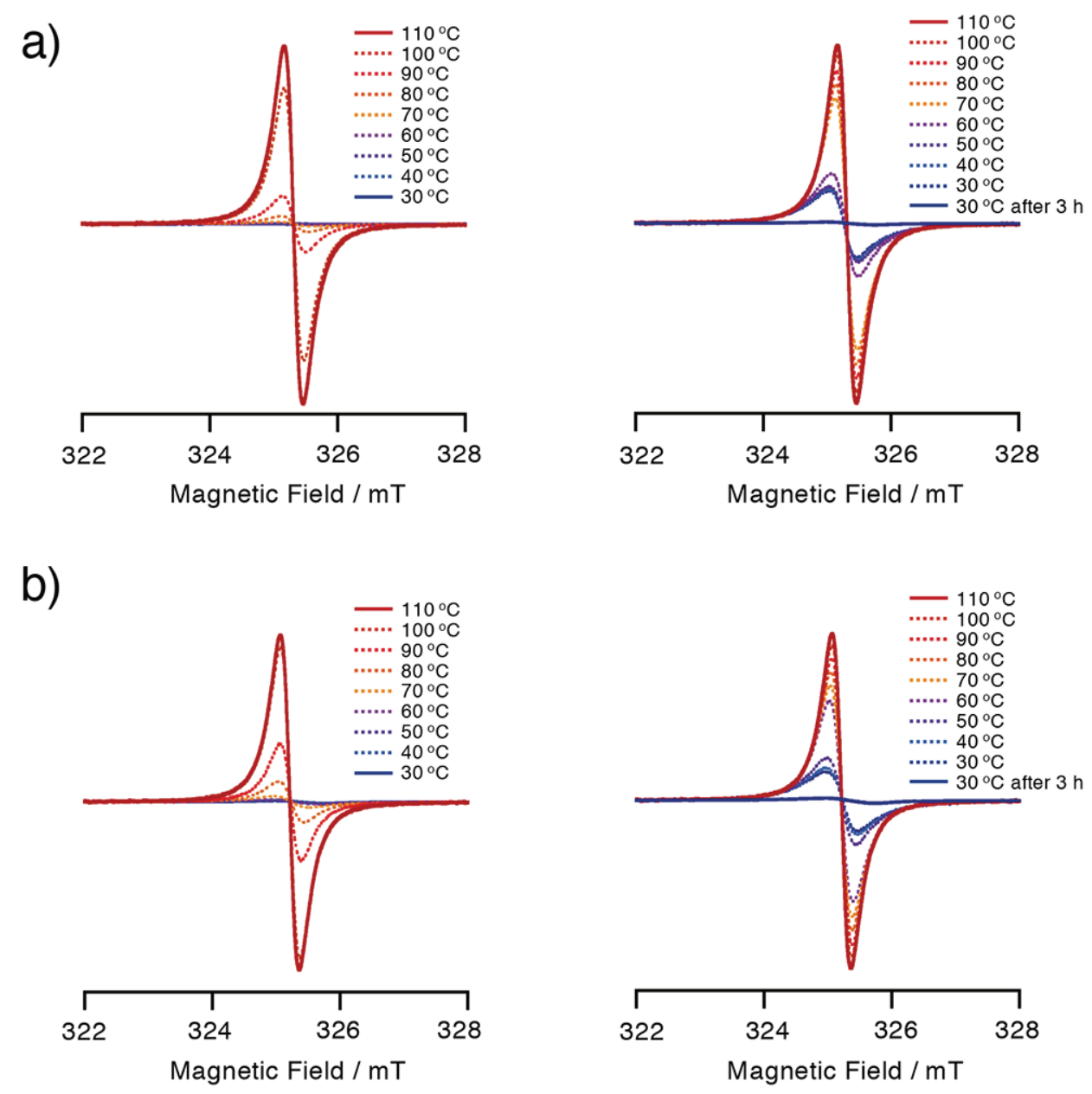

Figure S5. ESR spectra changes at variable temperature: (a) First and (b) second processes upon heating (left) and cooling (right).

The initial sample of the first process in the DM-GS state was prepared from the PM-OL state by cooling and standing for $3 \mathrm{~h}$ at $30{ }^{\circ} \mathrm{C}$. The ESR spectra were measured after reaching the corresponding temperature and standing for 2 minutes. The signal intensity increased significantly during the transition to the PM-OL state upon heating. Upon cooling, the signal intensity decreased during the transition to the PM-OS state around at $60-50{ }^{\circ} \mathrm{C}$. The PM-OS state at $50-30{ }^{\circ} \mathrm{C}$ remained ESR active, whereas the DM-GS state generated by standing for $3 \mathrm{~h}$ at $30{ }^{\circ} \mathrm{C}$ was ESR silent. The hysteretic changes in the ESR intensities were observed repeatedly as shown in the second process. 


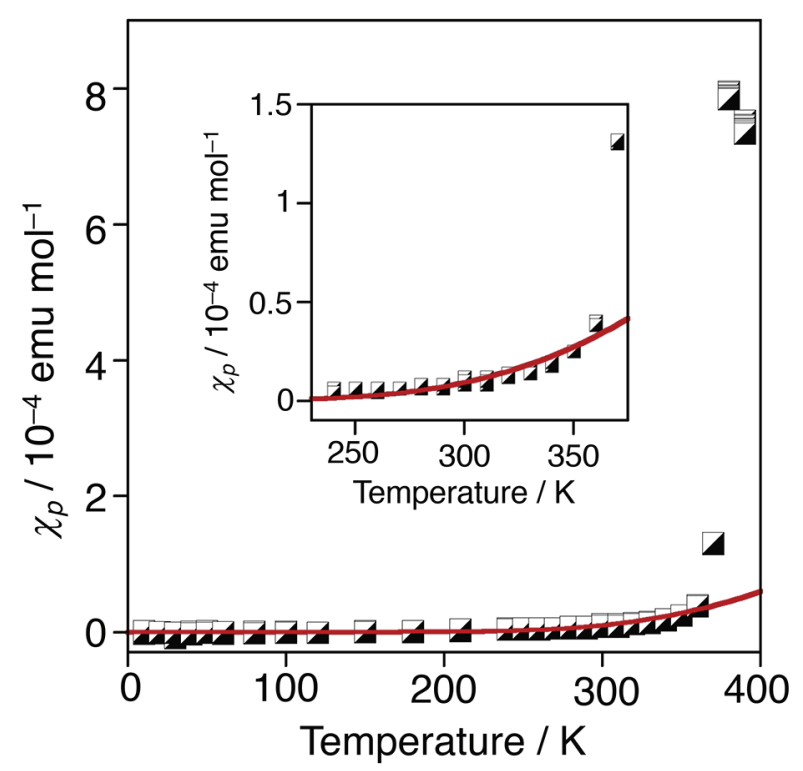

Figure S6. Temperature dependence of $\chi_{\mathrm{p}}$ for $\mathbf{1}^{\bullet+} \cdot \mathbf{N T f}_{2}^{-}$. The red line is simulated based on the singlettriplet model with $2 J / k_{\mathrm{B}}=-2.58(1) \times 10^{3} \mathrm{~K}$. Upon further heating above $370 \mathrm{~K}\left(97^{\circ} \mathrm{C}\right)$, the $\chi_{\mathrm{p}}$ value deviated from the estimated values obtained by the singlet-triplet model and increased drastically, indicating that the dimer structure collapsed to the monomer species. 
a)

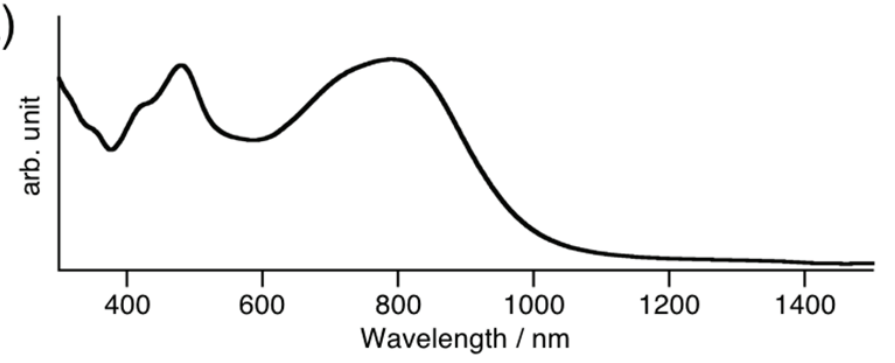

b)

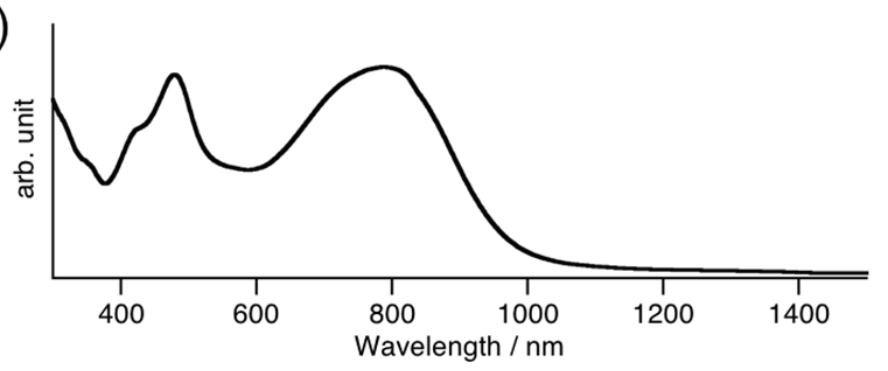

c)

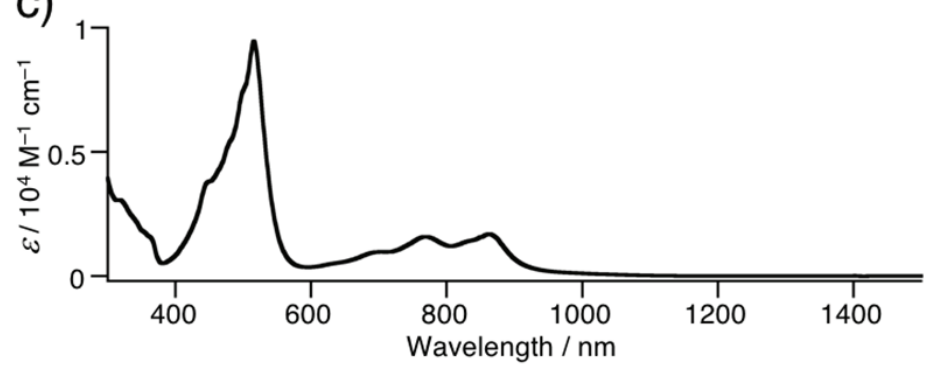

Figure S7. UV/vis/near-IR spectra in the (a) DM-GC and (b) DM-GS states using KBr pellets, and (c) in dichloromethane solution of $\mathbf{1}^{\mathbf{1}^{+} \cdot \mathbf{N T f}_{2}^{-}}$. 
a)

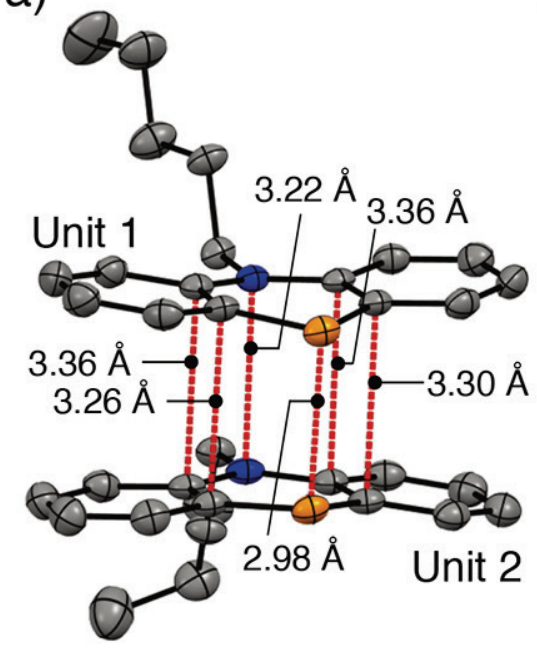

b)

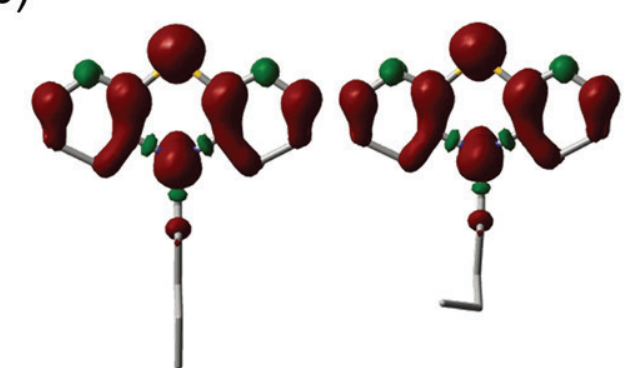

Unit 1

Unit 2

Positive spin density

Negative spin density

Figure S8. a) Detailed dimer structure of $\mathbf{1}^{\mathbf{*}}$ in the DM-GC state: The red dashed lines denote the short intermolecular contacts. The hydrogen atoms are omitted for clarity. b) Spin density distribution maps of optimized structures calculated from the geometry of the crystal structure by Gaussian 09 at the $u \omega b 97 x d / 6-31 g * *$ level of theory. ${ }^{\mathrm{S} 1}$ 


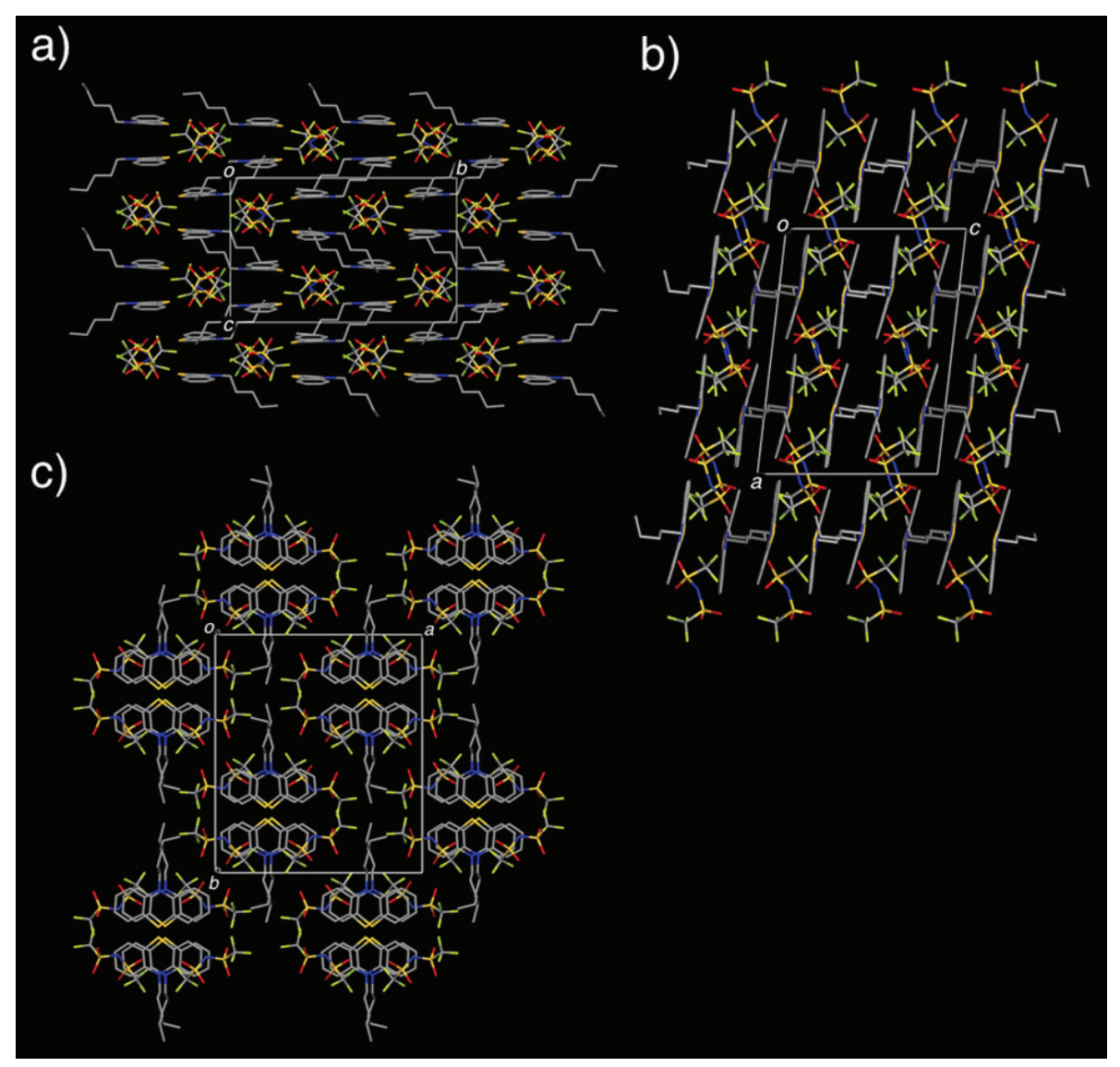

Figure S9. Perspective views of the packing structure for $\mathbf{1}^{\bullet+} \cdot \mathbf{N T f _ { 2 } ^ { - }}$ in the DM-GC state from the (a) $a$, (b) $b$, and (c) $c$ axis. 


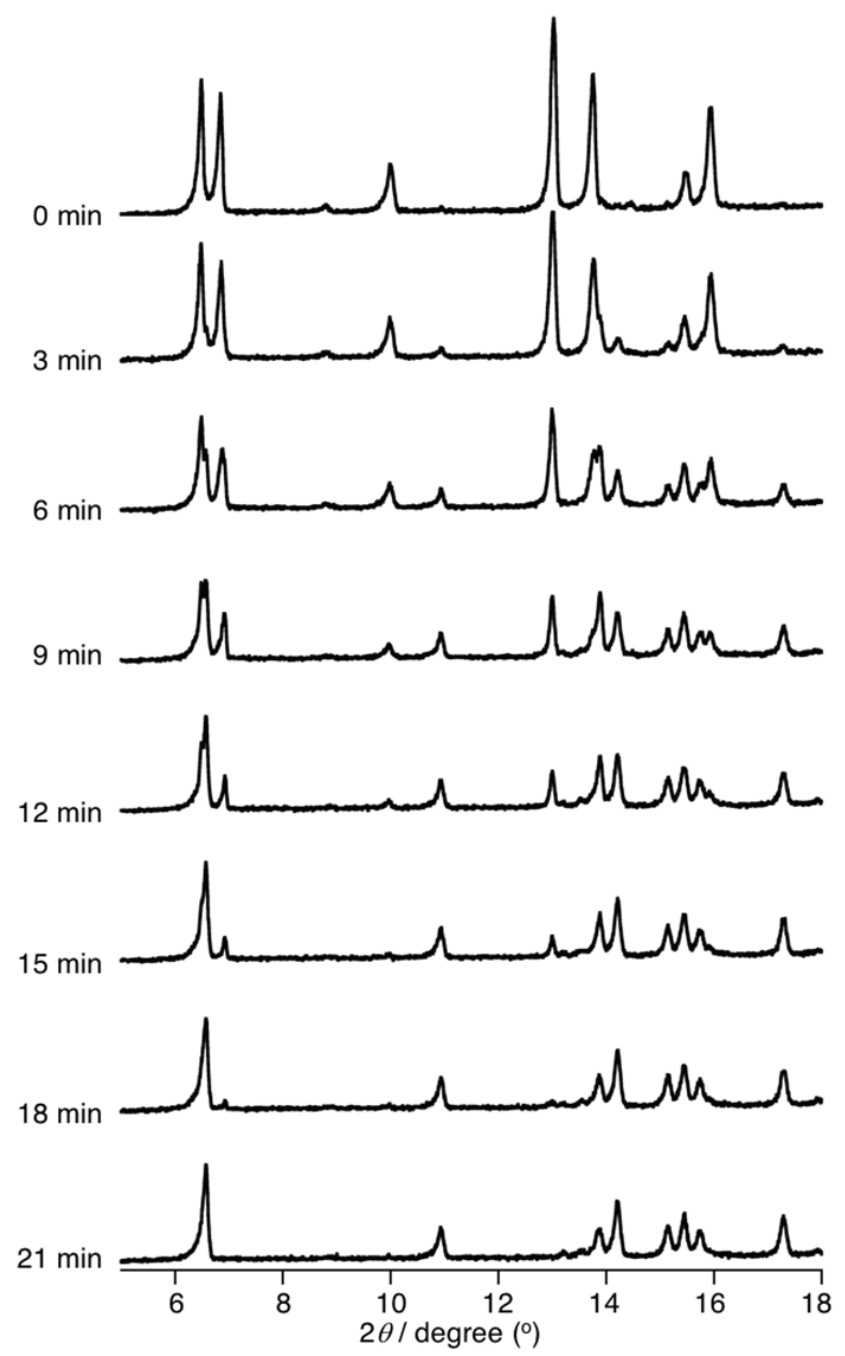

Figure S10. Powder XRD patterns at the phase transition between the PM-OS and the DM-GS states. 
a)

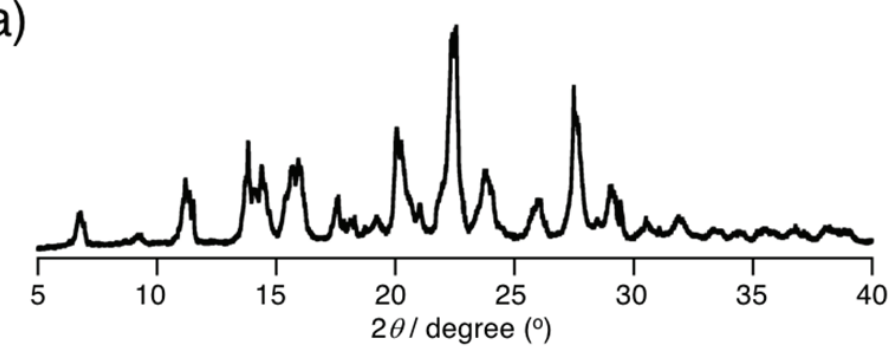

b)

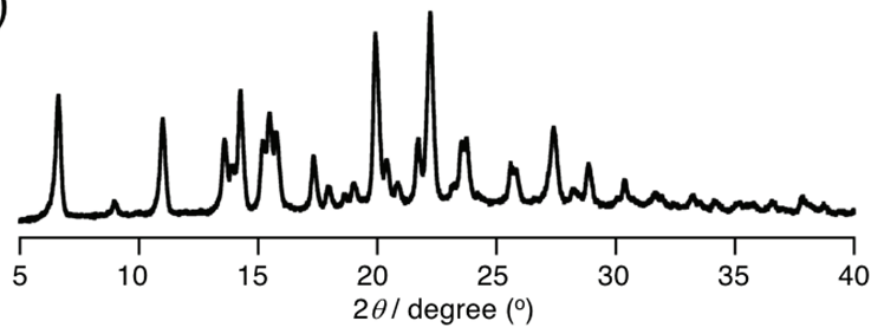

Figure S11. Powder XRD patterns in the DM-GC (a) and DM-GS (b) states. 


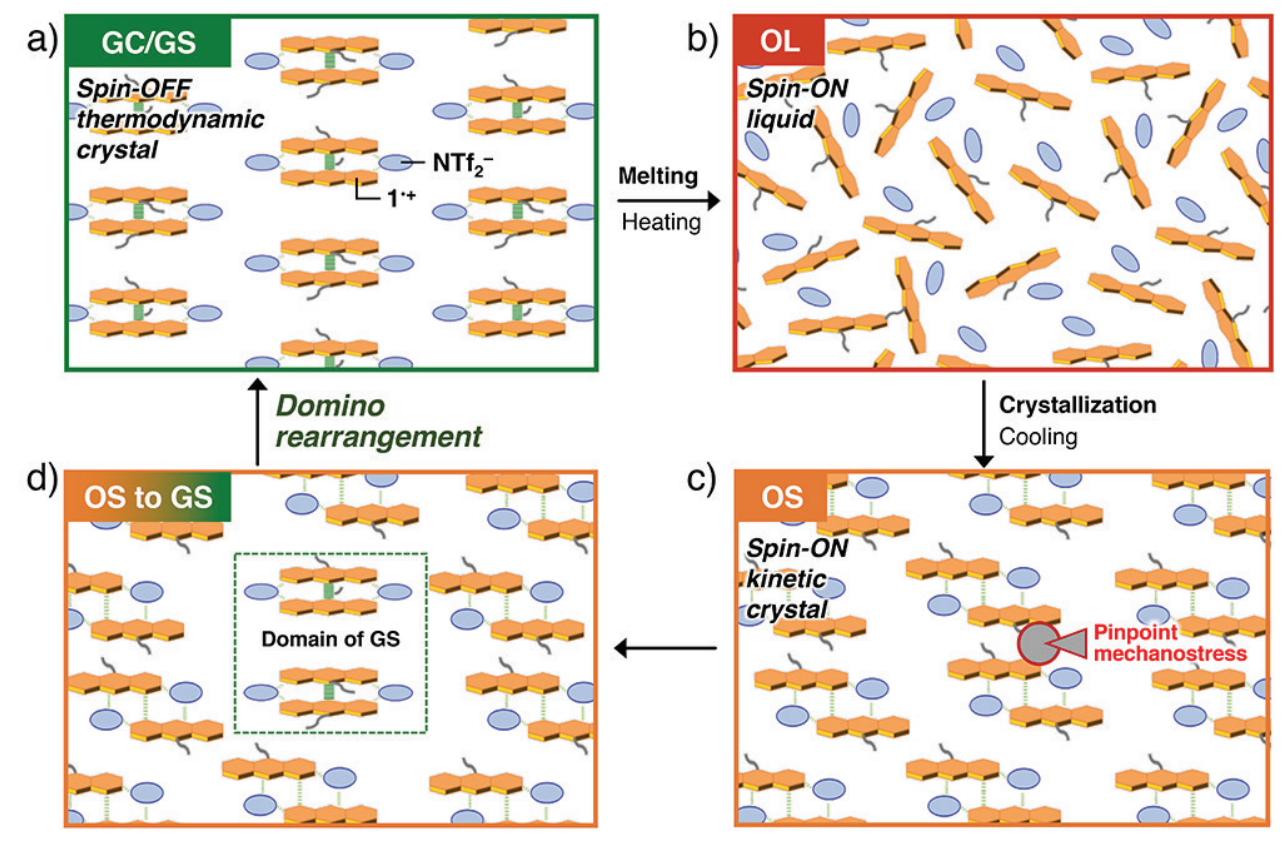

Figure S12. Proposed mechanism of the changes in radical associates with phase transitions. 
a)

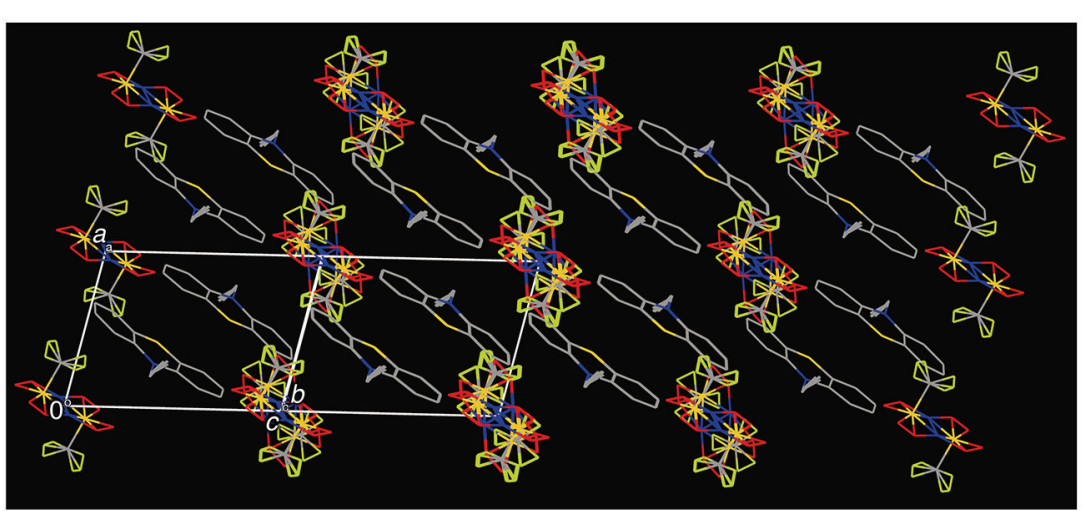

b)

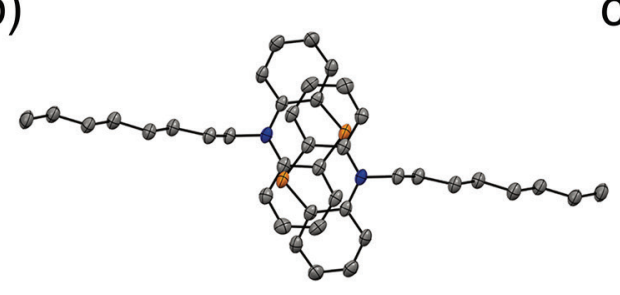

c)

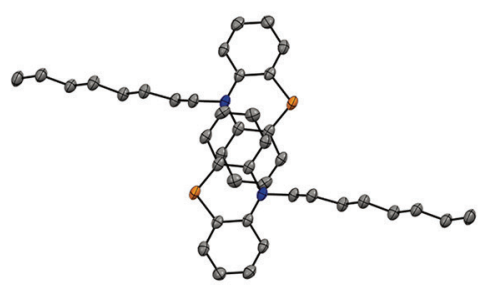

Figure S13. (a) Packing structure of the $N$-octyl analogue projected on the $(0 \overline{1} 1)$ plane. The radical cation forms pseudo-1D structure with a slipped stacking dimer structure. The nearest $\mathrm{C}-\mathrm{C}$ contact in the slipped stacking dimer is found to be $3.37 \AA$. The inter-planar distance of inter-dimer is slightly far from the sum of van der Waals radii of the carbon atoms (> $3.5 \AA$ A). (b) Slipped stacking dimer structure. (c) Packing structure of the inter-dimer motif. 

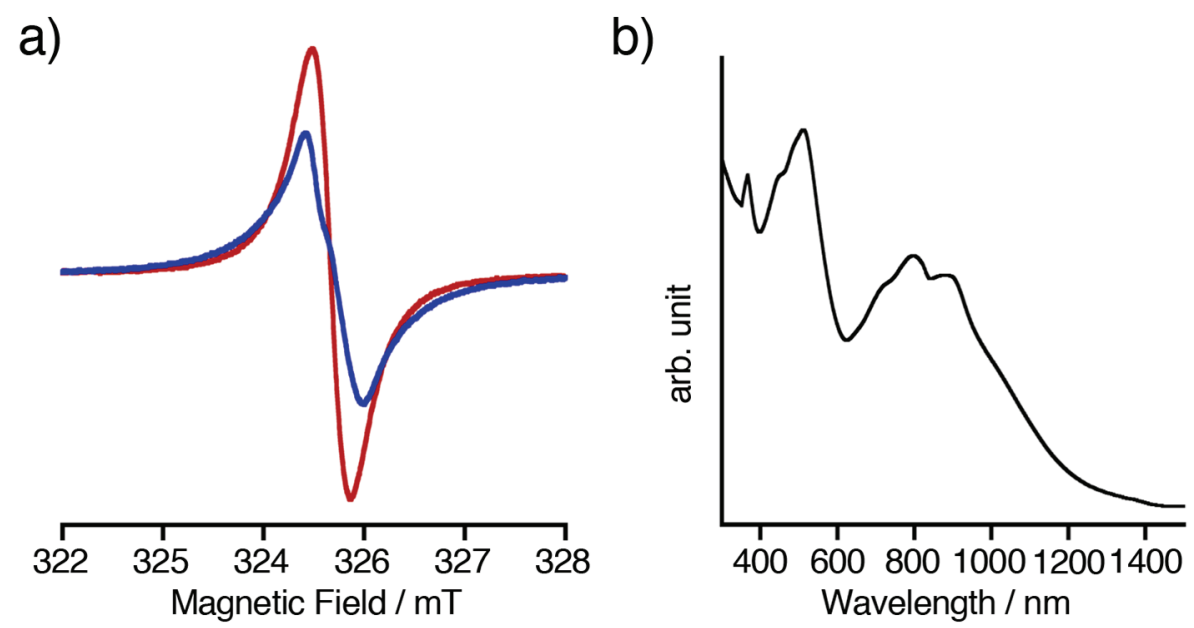

Figure S14. (a) ESR spectra of the $N$-octyl analogue at $30{ }^{\circ} \mathrm{C}$ (blue line) and $110{ }^{\circ} \mathrm{C}$ (red line), respectively. (b) UV/vis/near-IR spectrum of the $N$-octyl analogue using a KBr pellet. 
Table S1. Calculated total energies of high-spin (HS) and low-spin (LS) states, and the exchange interactions $\left(2\left(J_{\text {calc }} / k_{\mathrm{B}}\right) / \mathrm{K}\right)$ of the intermolecular contacts in the dimeric pairs of $\mathbf{1}^{\cdot+}$ and the $N$-octyl analogue..$^{[\mathrm{a}]}$

\begin{tabular}{ccccc}
\hline Dimeric pair $^{[\mathrm{b}]}$ & Spin state & $E /$ au & $<S^{2}>$ & $2\left(J_{\text {calc }} / k_{\mathrm{B}}\right) / \mathrm{K}$ \\
\hline $\mathbf{1}^{++}$ & HS $(S=1)$ & -2222.9742278 & 2.027705 & -5959 \\
& LS $(S=0)$ & -2222.9876713 & 0.603012 & \\
\hline \multirow{2}{*}{$N$-octyl analogue } & HS $(S=1)$ & -2458.7300762 & 2.032205 & -293 \\
& LS $(S=0)$ & -2458.7305466 & 1.01973 & \\
\hline
\end{tabular}

[a] The total energies were calculated by Gaussian 09 using density functional theory at uwb97xd/6$31 \mathrm{~g} * *$ level of theory. The total energy calculated using UHF-SCF procedure involving spin contamination due to the higher spin states. In order to eliminate the exchange interaction, a compensation equation, $2 J_{\text {calc }}=2\left({ }^{\mathrm{LS}} E-{ }^{\mathrm{HS}} E\right) /\left(<{ }^{\mathrm{HS}} S^{2}>-<{ }^{\mathrm{LS}} S^{2}>\right) \cdot{ }^{[\mathrm{S} 2]}[\mathrm{b}]$ Geometries were extracted from the X-ray structures (Figure S8, S13). 
a)

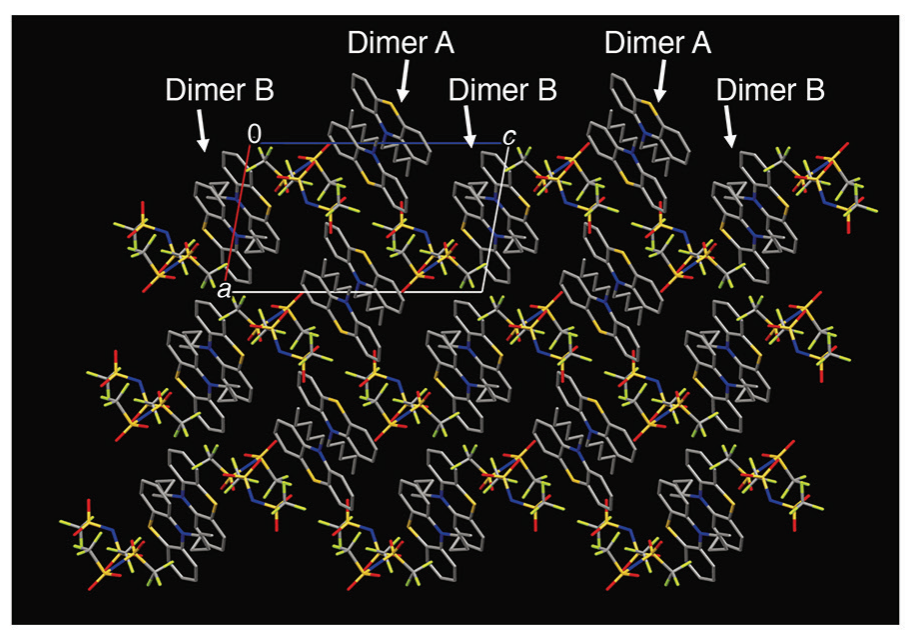

b)

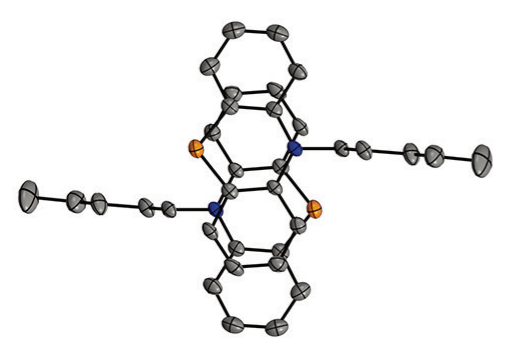

Dimer A c)

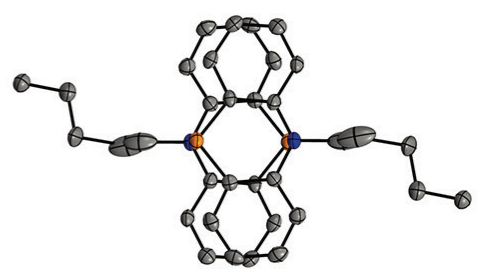

Dimer B

Figure S15. (a) Packing diagram and dimer structures in the polymorph crystal for $\mathbf{1}^{+\cdot} \cdot \mathbf{N T f}_{2}^{-}$. The polymorph crystals of $\mathbf{1}^{\cdot+} \cdot \mathbf{N T f}_{2}^{-}$imperceptibly were obtained as mixtures of large numbers of the DM-GC crystals by rapid recrystallization from dichloromethane/diethyl ether. In the packing diagram, two kinds of dimer structures, Dimer A and B, were observed: (b) Dimer A: Slipped stacking dimer structure (C-S contact: 3.36 ̊̊). (c) Dimer B: Co-facial $\pi$-dimer structure (N-S contact: 3.29 $\AA$ ). The polymorph crystals showed unprecedented phase transitions to the same crystal structure in the DM-GC by single-crystal-single-crystal transformations after heating at $80^{\circ} \mathrm{C}$ for several minutes. Accompanying with the transformation, all of the dimers with head-to-tail manner changed to the dimers with head-to-head motifs. See, supporting cif files (CCDC 1915034 before heating, and CCDC 1917469 after heating) and Table S2. 
a)

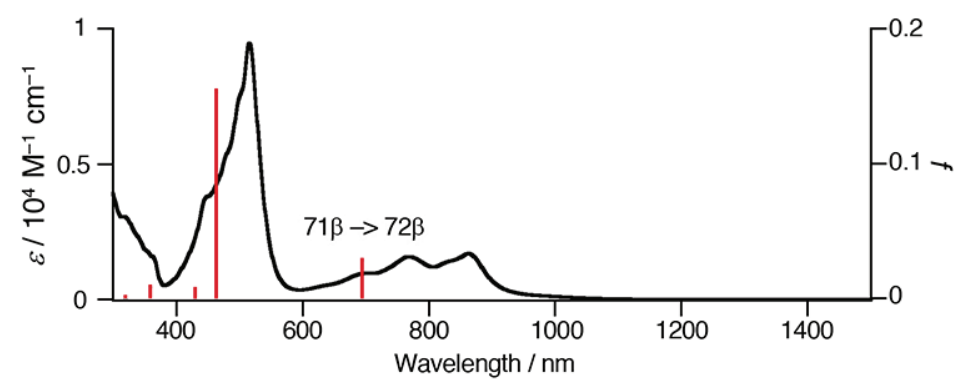

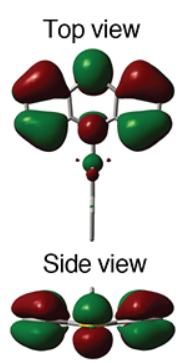

$71 \beta($ NHOMO- $\beta)$

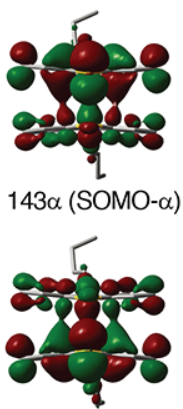

$143 \beta($ SOMO- $\beta$ )

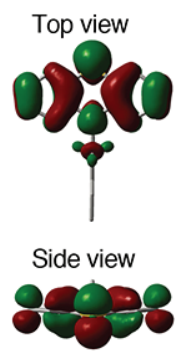

$72 \beta(\mathrm{SOMO}-\beta)$

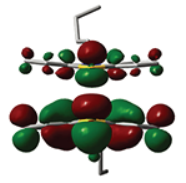

$144 \alpha($ LUMO- $\alpha)$

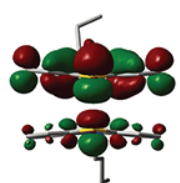

$144 \beta($ LUMO- $\beta$ )

Figure S16. UV/vis/near-IR spectra in (a) dichloromethane solution and (b) the DM-GC state (KBr pellet) of $\mathbf{1}^{\cdot+} \cdot \mathbf{N T f}_{\mathbf{2}}^{-}$with the results of TDDFT calculations (red bar, Gaussian 09 using density functional theory at $\mathrm{u} \omega \mathrm{b} 97 \mathrm{xd} / 6-31 \mathrm{~g} * *$ level of theory). The contributing molecular orbitals for the longest absorption bands were shown in the right side. 
Table S2. Crystallographic data of $\mathbf{1}^{\bullet+} \cdot \mathbf{N T f}_{\mathbf{2}}{ }^{-}$and the $N$-octyl analogue

\begin{tabular}{|c|c|c|c|c|}
\hline & $1^{{ }^{+} \bullet \mathrm{NTf}_{2}^{-}}$ & $N$-Octyl analogue & $\begin{array}{l}\text { Polymorph of } \\
\mathbf{1}^{\bullet+} \cdot \mathbf{N T f}_{2}^{-}\end{array}$ & $\begin{array}{l}\text { Polymorph of } \mathbf{1}^{\cdot+} \cdot \mathbf{N T f}_{\mathbf{2}}^{-} \\
\text {after heating at } 80{ }^{\circ} \mathrm{C}\end{array}$ \\
\hline CCDC number & 1846261 & 1901287 & 1915034 & 1917469 \\
\hline Formula & $\mathrm{C}_{19} \mathrm{H}_{19} \mathrm{~F}_{6} \mathrm{~N}_{2} \mathrm{O}_{4} \mathrm{~S}_{3}$ & $\mathrm{C}_{22} \mathrm{H}_{25} \mathrm{~F}_{6} \mathrm{~N}_{2} \mathrm{O}_{4} \mathrm{~S}_{3}$ & $\mathrm{C}_{19} \mathrm{H}_{19} \mathrm{~F}_{6} \mathrm{~N}_{2} \mathrm{O}_{4} \mathrm{~S}_{3}$ & $\mathrm{C}_{19} \mathrm{H}_{19} \mathrm{~F}_{6} \mathrm{~N}_{2} \mathrm{O}_{4} \mathrm{~S}_{3}$ \\
\hline Formula weight & 549.54 & 591.62 & 549.54 & 549.54 \\
\hline Crystal color, morphology & green, block & orange, platelet & green, platelet & green, platelet \\
\hline Crystal size $/ \mathrm{mm}^{3}$ & $0.20 \times 0.20 \times 0.20$ & $0.40 \times 0.20 \times 0.02$ & $0.30 \times 0.08 \times 0.02$ & $0.30 \times 0.08 \times 0.02$ \\
\hline Crystal system & monoclinic & triclinic & triclinic & monoclinic \\
\hline Space group & $P 2_{1} / c(\# 14)$ & $P-1(\# 2)$ & $P-1(\# 2)$ & $P 2_{1} / c(\# 14)$ \\
\hline$a / \AA$ & $17.677(2)$ & $7.6830(15)$ & $9.5649(14)$ & $17.687(3)$ \\
\hline$b / \AA$ & $20.167(3)$ & $13.097(3)$ & $14.900(2)$ & $20.208(3)$ \\
\hline$c / \AA$ & $12.8973(16)$ & $13.447(2)$ & $16.507(3)$ & 12.9161(19) \\
\hline$\alpha /$ degree & 90 & $82.425(15)$ & $97.926(5)$ & 90 \\
\hline$\beta /$ degree & $96.700(3)$ & $110.369(8)$ & $99.466(4)$ & $96.737(4)$ \\
\hline$\gamma /$ degree & 90 & $76.579(14)$ & $95.106(3)$ & 90 \\
\hline$V / \AA^{3}$ & $4566.4(10)$ & $1278.6(4)$ & $2283.3(6)$ & $4584.6(12)$ \\
\hline$Z$ value & 8 & 2 & 4 & 8 \\
\hline$T / \mathrm{K}$ & $150(2)$ & $150(2)$ & $150(2)$ & $150(2)$ \\
\hline$D_{\text {calc }} / \mathrm{g} \mathrm{cm}^{-3}$ & 1.599 & 1.537 & 1.599 & 1.592 \\
\hline$F(000)$ & 2248.00 & 610.00 & 1124.00 & 2248.00 \\
\hline$\mu / \mathrm{cm}^{-1}$ & 40.3 & 36.6 & 40.3 & 40.2 \\
\hline No. of reflections measured & 42448 & 23750 & 42413 & 42861 \\
\hline No. of unique reflections & 10335 & 5822 & 10411 & 10531 \\
\hline No. of observed reflections & 10391 & 5687 & 10411 & 10531 \\
\hline No. of variables & 613 & 433 & 613 & 613 \\
\hline Reflection/Parameter Ratio & 17.0 & 13.1 & 17.0 & 17.2 \\
\hline$R 1[I>2.00 \sigma(I)]$ & 0.0510 & 0.0572 & 0.0614 & 0.0632 \\
\hline$R_{\mathrm{w}}$ & 0.1299 & 0.1257 & 0.1648 & 0.1370 \\
\hline Goodness-of-fit & 1.090 & 0.837 & 1.025 & 0.956 \\
\hline
\end{tabular}




\section{References}

(S1) Gaussian 09, Revision D.01, Frisch, M. J.; Trucks, G. W.; Schlegel, H. B.; Scuseria, G. E.; Robb, M. A.; Cheeseman, J. R.; Scalmani, G.; Barone, V.; Mennucci, B.; Petersson, G. A.; Nakatsuji, H.; Caricato, M.; Li, X.; Hratchian, H. P.; Izmaylov, A. F.; Bloino, J.; Zheng, G.; Sonnenberg, J. L.; Hada, M.; Ehara, M.; Toyota, K.; Fukuda, R.; Hasegawa, J.; Ishida, M.; Nakajima, T.; Honda, Y.; Kitao, O.; Nakai, H.; Vreven, T.; Montgomery, J. A., Jr.; Peralta, J. E.; Ogliaro, F.; Bearpark, M.; Heyd, J. J.; Brothers, E.; Kudin, K. N.; Staroverov, V. N.; Kobayashi, R.; Normand, J.; Raghavachari, K.; Rendell, A.; Burant, J. C.; Iyengar, S. S.; Tomasi, J.; Cossi, M.; Rega, N.; Millam, J. M.; Klene, M.; Knox, J. E.; Cross, J. B.; Bakken, V.; Adamo, C.; Jaramillo, J.; Gomperts, R.; Stratmann, R. E.; Yazyev, O.; Austin, A. J.; Cammi, R.; Pomelli, C.; Ochterski, J. W.; Martin, R. L.; Morokuma, K.; Zakrzewski, V. G.; Voth, G. A.; Salvador, P.; Dannenberg, J. J.; Dapprich, S.; Daniels, A. D.; Farkas, Ö.; Foresman, J. B.; Ortiz, J. V.; Cioslowski, J.; Fox, D. J. Gaussian, Inc., Wallingford CT, 2009.

(S2) Yamaguchi, K.; Kawakami, T.; Takano, Y.; Kitagawa, Y.; Yamashita, Y.; Fujita, H. Int. J. Quantum Chem. 2002, 90, 370. 